\title{
Alogliptin alleviates hepatic steatosis in a mouse model of nonalcoholic fatty liver disease by promoting CPT1a expression via Thr172 phosphorylation of AMPK $\alpha$ in the liver
}

\author{
HIROSHI TOBITA, SHUICHI SATO, TOMOTAKA YAZAKI, TSUYOSHI MISHIRO, \\ NORIHISA ISHIMURA, SHUNNJI ISHIHARA and YOSHIKAZU KINOSHITA
}

Department of Gastroenterology and Hepatology, Shimane University Faculty of Medicine, Izumo, Shimane 693-8501, Japan

Received November 14, 2017; Accepted February 6, 2018

DOI: $10.3892 / \mathrm{mmr} .2018 .8673$

\begin{abstract}
Pioglitazone (PIO) has been reported to be effective for nonalcoholic fatty liver disease (NAFLD) and alogliptin (ALO) may have efficacy against NAFLD progression in patients with type 2 diabetes mellitus (T2DM). The present study examined the effectiveness of ALO in a rodent model of NAFLD and diabetes mellitus. KK-A ${ }^{\mathrm{y}}$ mice were used to produce an NAFLD model via administration of a choline-deficient (CD) diet. To examine the effects of alogliptin, KK-A $\mathrm{A}^{\mathrm{y}}$ mice were provided with a $\mathrm{CD}$ diet with $0.03 \% \mathrm{ALO}$ and/or $0.02 \% \mathrm{PIO}$ orally for 8 weeks. Biochemical parameters, pathological alterations and hepatic mRNA levels associated with fatty acid metabolism were assessed. Severe hepatic steatosis was observed in $\mathrm{KK}-\mathrm{A}^{\mathrm{y}}$ mice fed with a CD diet, which was alleviated by the administration of ALO and/or PIO. ALO administration increased the hepatic carnitine palmitoyltransferase 1a (CPT1a) mRNA expression level and enhanced the Thr172 phosphorylation of AMP-activated protein kinase $\alpha(\mathrm{AMPK} \alpha)$ in the liver. PIO administration tended to decrease the hepatic fatty acid synthase mRNA expression level and increase the serum adiponectin level. Homeostasis model of assessment-insulin resistance values tended to improve with ALO and PIO administration. ALO and PIO alleviated hepatic steatosis in $\mathrm{KK}-\mathrm{A}^{\mathrm{y}}$ mice fed with a CD diet. ALO increased hepatic mRNA expression levels associated with fatty acid oxidation. In addition, the results of the present study suggested that ALO promotes CPT1a expression via Thr172 phosphorylation of AMPK $\alpha$.
\end{abstract}

Correspondence to: Dr Hiroshi Tobita, Department of Gastroenterology and Hepatology, Shimane University Faculty of Medicine, 89-1 Enya-cho, Izumo, Shimane 693-8501, Japan E-mail: ht1020@med.shimane-u.ac.jp

Key words: nonalcoholic fatty liver disease, diabetes mellitus, alogliptin, carnitine palmitoyltransferase 1a, AMP-activated protein kinase $\alpha, \mathrm{KK}^{\mathrm{y}} \mathrm{A}^{\mathrm{y}}$ mice

\section{Introduction}

Nonalcoholic fatty liver disease (NAFLD) is the most common chronic liver disease with a worldwide with a prevalence ranging from 6 to $35 \%$ (1). In patients with type 2 diabetes mellitus (T2DM), the prevalence of NAFLDis $60 \%$ (2). Patients with NAFLD are at a high risk of mortality from cardiovascular disease and liver-associated diseases, including cirrhosis complications and hepatocellular carcinoma (3). In particular, patients with NAFLD and T2DM are at risk of liver-associated mortality (4); however, at present, no pharmacological therapies are established for T2DM patients with NAFLD refractory to lifestyle intervention. Thiazolidinediones (TZDs) and dipeptidyl peptidase-4 (DPP-4) inhibitors are known to be effective for the treatment of various T2DM pathophysiological processes. Pioglitazone (PIO), which is one of the TZDs, has been demonstrated to decrease serum liver enzyme levels, and to improve hepatic steatosis and lobular inflammation in patients with nonalcoholic steatohepatitis (NASH) without T2DM (5). PIO additionally prevented methionine- and choline-deficient (CD) diet-induced steatohepatitis in mice (6). Among the available DPP-4 inhibitors, sitagliptin is reported to reduce serum liver enzyme levels and decrease ballooning score in patients with NASH and T2DM (7). Alogliptin (ALO) may possess efficacy against NAFLD progression in patients with T2DM (8). However, whether ALO is effective for NAFLD remains to be elucidated. The present study examined the effectiveness of ALO in a rodent model of NAFLD and diabetes mellitus.

\section{Materials and methods}

Animal experiments. PIO and ALO were provided by Takeda Pharmaceutical Company, Ltd. (Osaka, Japan). The CD diet and standard chow were purchased from Oriental Yeast Co., Ltd. (Tokyo, Japan). Eight-week-old 30 male KK-A ${ }^{\mathrm{y}}$ mice were purchased from CLEA Japan, Inc. (Tokyo, Japan). These mice demonstrate ectopic overexpression of agouti peptide, an endogenous melanocortin-4 receptor (MC4R) antagonist, and social isolation promotes obesity due primarily to decreased energy expenditure and secondarily to increased food consumption. In addition, such isolation leads to insulin-independent diabetes associated with increased hepatic gluconeogenic 
gene expression (9). KK-A $\mathrm{A}^{\mathrm{y}}$ mice were housed individually in stainless-steel cages, and provided with unrestricted access to chow and tap water throughout the duration of the present study. KK-Ay mice were housed in the room of $23 \pm 3^{\circ} \mathrm{C}$ and $50 \pm 10 \%$ humidity and maintained on $12 \mathrm{~h} \mathrm{light/dark} \mathrm{cycle.}$ Following a 4 -week acclimation period (12 weeks of age), the mice $(45.5 \pm 4.9 \mathrm{~g})$ were randomly separated into five groups of 6 mice each and administered the following diets: Control group, standard chow; CD group, a CD diet; PIO group, the $\mathrm{CD}$ diet containing $0.02 \%$ PIO; ALO group, the CD diet containing $0.03 \%$ ALO; and the PIO+ALO group, the CD diet with $0.02 \%$ PIO and $0.03 \%$ ALO. Following 8 weeks of the diet, the mice received no food for $18 \mathrm{~h}$ and were administered ether anesthesia and euthanized by exsanguination from the inferior vena cava. Serum and hepatic tissue samples were obtained and frozen at $-80^{\circ} \mathrm{C}$ until assayed. Certain hepatic tissues were fixed in $10 \%$ formalin for $48 \mathrm{~h}$ at room temperature and $3 \mu \mathrm{m}$ paraffin-embedded hepatic tissues were stained with hematoxylin for $10 \mathrm{~min}$ and eosin for $5 \mathrm{~min}$ at room temperature. Then the histology of samples was analyzed under a light microscope (magnification, $\mathrm{x} 40$ or $\mathrm{x} 100$ ). The present study was performed in strict accordance with the recommendations in the Guide for the Care and Use of Laboratory Animals of the National Institutes of Health (Bethesda, MD, USA). The protocol was approved by the Institute for Animal Experimentation of Shimane University (Shimane, Japan; protocol no. IZ23-164).

Measurements of serum parameters. Serum levels of aspartate aminotransferase (AST), alanine aminotransferase (ALT), $\gamma$-glutamyl transpeptidase $(\gamma$-GTP), triglyceride $(\mathrm{TG})$, total cholesterol (T-Chol) and high-density lipoprotein-cholesterol (HDL-C), in addition to fasting serum glucose were measured using a Spotchem 4430 Benchtop Biochemistry Analyzer (Arkray; SCIL, Holtzheim, France). Serum levels of fasting insulin and adiponectin were measured using ELISA kits (AKRIN-011T and AKMAN-011, respectively; Shibayagi Co., Ltd., Tokyo, Japan). Homeostasis model of assessment-insulin resistance (HOMA-IR) values were calculated using the following formula: HOMA-IR=[fasting serum glucose $(\mathrm{mmol} / \mathrm{l}) \mathrm{x}$ fasting serum insulin $(\mathrm{ng} / \mathrm{ml})] / 22.5(10)$.

Determination of hepatic mRNA levels. Total RNA was isolated from hepatic tissues with RNAlater RNA Stabilization Reagent and purified using an RNeasy Mini kit (both from Qiagen GmbH, Hilden, Germany). Samples of cDNA were generated using a cDNA synthesis kit according to the manufacturer's protocols (Applied Biosystems; Thermo Fisher Scientific, Inc., Waltham, MA, USA). RT-qPCR was then performed using an ABI PRISM 7700 sequence detection system (Thermo Fisher Scientific, Inc.) with SYBR ${ }^{\circledR}$-Green PCR master mix (Applied Biosystems; Thermo Fisher Scientific, Inc.). The PCR protocol used was as follows: Enzyme activation: $94^{\circ} \mathrm{C}$ for $10 \mathrm{~min}$; thermocycling, $94^{\circ} \mathrm{C}$ for $15 \mathrm{sec}, 60^{\circ} \mathrm{C}$ for $1 \mathrm{~min}$, repeat for 43 cycles. The relative expression of each gene was normalized to that of GAPDH. qPCR was replicated two times for each sample of cDNA. Primers for mouse fatty acid transport protein (FATP)1-4, fatty acid synthase (FAS), liver-type fatty acid binding protein (L-FABP), acyl-coenzyme A oxidase 1 (AOX) and long-chain acyl-coenzyme A dehydrogenase (LCAD) genes were prepared as previously described (11). Primers for mouse peroxisome proliferator-activated receptor $\alpha(\operatorname{PPAR} \alpha), \alpha$ smooth muscle actin ( $\alpha$ SMA), superoxide dismutase 1 (SOD1), carnitine palmitoyltransferase 1a (CPT1a), microsomal triglyceride transfer protein (MTP), monocyte chemoattractant protein 1 (MCP1) and GAPDH genes were prepared using NCBI blast tool and Primer3 Input (version 0.4.0, http://bioinfo.ut.ee/ primer3-0.4.0/) which is most commonly used for primer design (12). The primers are presented in Table I. All the mRNA levels were normalized to GAPDH mRNA in the same preparation.

Protein extraction and western blotting assays. Protein extraction was performed using PRO-PREP ${ }^{\mathrm{TM}}$ protein extraction solution (Intron Biotechnology, Inc., Seongnam, Korea), according to the manufacturer's protocol. A total of $10 \mathrm{mg}$ of liver tissue was suspended in $500 \mu 1$ PRO-PREP ${ }^{\mathrm{TM}}$ solution mixed with $5 \mu$ l Phosphatase-Inhibitor Mix II (Cosmo Bio Co., Ltd., Tokyo, Japan). Subsequently, the tissue was disrupted using a Tissue Lyser II (Qiagen $\mathrm{GmbH}$ ) for $3 \mathrm{~min}$ at $30 \mathrm{~Hz}$ and homogenized with a syringe equipped with a 23-G needle, followed by incubation on ice for $30 \mathrm{~min}$ and sonication with Bioruptor UCD-200 TM (Cosmo Bio Co., Ltd.). In water cooled with ice, sonication for $5 \mathrm{~min}$ at $20 \mathrm{KHz}$ was performed for $30 \mathrm{sec}$ every $60 \mathrm{sec}$. The resulting liver tissue lysate was centrifuged at $14,000 \mathrm{x}$ g for $5 \mathrm{~min}$ at $4^{\circ} \mathrm{C}$, and the supernatant was collected and subjected to downstream processing. The protein concentration was estimated using a Pierce $^{\mathrm{TM}}$ Bicinchoninic Acid Protein Assay kit (Takara Bio, Inc., Otsu, Japan), for which $50 \mu \mathrm{g}$ protein/lane was loaded and processed for SDS-PAGE fractionation (4-12\% SDS-PAGE mini; Tefco Technical Frontier Co., Tokyo, Japan) and transferred to a polyvinylidene difluoride membrane (Hybond-P; GE Healthcare Life Sciences, Little Chalfont, UK). Following blocking of the membrane for $1 \mathrm{~h}$ using $5 \%$ skimmed milk (Difco; BD Biosciences, Franklin Lakes, NJ, USA) in TBS-Tween-20 (TBST; TBS and 0.05\% Tween-20, pH 7.4) at room temperature, it was incubated for $24 \mathrm{~h}$ with a phosphorylated-AMP-activated protein kinase (AMPK) $\alpha$ (Thr172) rabbit monoclonal antibody (cat. no. 2535S; Cell Signaling Technology, Inc., Danvers, MA, USA) at a 1:500 dilution at $4^{\circ} \mathrm{C}$, reacted with peroxidase-conjugated polyclonal goat anti-rabbit immunoglobulins (cat. no. P7-0448; Dako; Agilent Technologies Inc., Santa Clara, CA, USA) at a 1:1,000 dilution at room temperature for $2 \mathrm{~h}$, and washed three times in TBST. The resulting signals were imaged using electrochemiluminescence (GE Healthcare Bio-Sciences, Pittsburgh, PA, USA). Goat anti- $\beta$-actin antibody (catalogue no. SC-47778; Santa Cruz Biotechnology, Inc., Dallas, TX, USA) was used as an internal control. Band densitometry was performed using ImageJ software of version 1.51 (National Institutes of Health, Bethesda, MD, USA).

Statistical analysis. All data are presented as the mean \pm standard deviation $(n=3)$. Mann-Whitney's $U$ test was used for comparisons between two groups, while Steel-Dwass test following a Kruskal-Wallis test was used for comparisons among multiple groups. $\mathrm{P}<0.05$ was considered to indicate a statistically significant difference. All statistical analyses were 
Table I. Primer sequences for reverse transcription-quantitative polymerase chain reaction.

\begin{tabular}{|c|c|}
\hline Primer & Sequence \\
\hline FATP1-fwd & 5'-CGCTTTCTGCGTATCGTCTG-3' \\
\hline FATP1-rev & 5'-GATGCACGGGATCGTGTCT-3' \\
\hline FATP2-fwd & 5'-GGTATGGGACAGGCCTTGCT-3' \\
\hline FATP2-rev & 5'-GGGCATTGTGGTATAGATGACATC-3' \\
\hline FATP3-fwd & 5'-AGTGCCAGGGATTCTACCATC-3' \\
\hline FATP3-rev & 5'-GAACTTGGGTTTCAGCACCAC-3' \\
\hline FATP4-fwd & 5'-GATGGCCTCAGCTATCTGTGA-3' \\
\hline FATP4-rev & 5'-GGTGCCCGATGTGTAGATGTA-3' \\
\hline FAS-fwd & 5'-TGCTCCCAGCTGCAGGC-3' \\
\hline FAS-rev & 5'-GCCCGGTAGCTCTGGGTGTA-3' \\
\hline L-FABP-fwd & 5'-GTGGTCCGCAATGAGTTCAC-3' \\
\hline L-FABP-rev & 5'-GTATTGGTGATTGTGTCTCC-3' \\
\hline AOX-fwd & 5'-CTTGTTCGCGCAAGTGAGG-3' \\
\hline AOX-rev & 5'-CAGGATCCGACTGTTTACC-3' \\
\hline LCAD-fwd & 5'-AAGGATTTATTAAGGGCAAGAAGC-3 \\
\hline LCAD-rev & 5'-GGAAGCGGAGGCGGAGTC-3' \\
\hline PPAR $\alpha$-fwd & 5'-TGCAAACTTGGACTTGAACG-3' \\
\hline PPAR $\alpha-$ rev & 5'-TGATGTCACAGAACGGCTTC-3' \\
\hline aSMA-fwd & 5'-CAACTGGGACGACATGGAA-3' \\
\hline$\alpha$ SMA-rev & 5'-GGTCTCAAACATAATCTGGGTCA-3' \\
\hline SOD1-fwd & 5'-GAACCATCCACTTCGAGCAG-3' \\
\hline SOD1-rev & 5'-AAAATGAGGTCCTGCACTGG-3' \\
\hline CPT1a-fwd & 5'-TGTCAAAGATACCGTGAGCAG-3' \\
\hline CPT1a-rev & 5'-GCCCACCAGGATTTTAGCTT-3' \\
\hline MTP-fwd & 5'-CATGTCAGCCATCCTGTTTG-3' \\
\hline MTP-rev & 5'-CTCGCGATACCACAGACTGA-3' \\
\hline MCP1-fwd & 5'-AGGTCCCTGTCATGCTTCTG-3' \\
\hline MCP1-rev & 5'-TCATTGGGATCATCTTGCTG-3' \\
\hline GAPDH-fwd & 5'-ACCCAGAAGACTGTGGATGG-3' \\
\hline GAPDH-rev & 5'-GGTCCTCAGTGTAGCCCAAG-3' \\
\hline
\end{tabular}

FATP, fatty acid transport protein; FAS, fatty acid synthase; L-FABP, long-chain acyl-coenzyme A dehydrogenase; AOX, acyl-coenzyme A oxidase 1; LCAD, long-chain acyl-coenzyme A dehydrogenase; PPAR $\alpha$, peroxisome proliferator-activated receptor $\alpha ; \alpha$ SMA, $\alpha$ smooth muscle actin; SOD1, superoxide dismutase 1; CPT1a, carnitine palmitoyltransferase 1a; MTP, microsomal triglyceride transfer protein; MCP1, monocyte chemoattractant protein 1; GAPDH, glyceralaldehyde-3-phosphate dehydrogenase; fwd, forward; rev, reverse;

performed using Statistical Analysis Software (BellCurve for Excel, version 2.14; Social Survey Research Information Co., Ltd., Tokyo, Japan).

\section{Results}

Diet consumption and body weight are not affected by the administration of PIO or ALO. The Control group consumed a greater amount of food compared with the CD group (23.5 \pm 1.6 and $16.8 \pm 2.5 \mathrm{kcal} /$ body/day, respectively; $\mathrm{P}=0.006)$. No significant differences were identified in the amounts of food consumed among the CD, PIO, ALO and PIO+ALO groups (Table II). In the $\mathrm{CD}, \mathrm{PIO}, \mathrm{ALO}$ and PIO+ALO groups, mean body weights prior to starting the diet were $49.0 \pm 1.4,48.2 \pm 2.4,48.5 \pm 2.5$, $48.2 \pm 2.3$ and $48.9 \pm 2.3 \mathrm{~g}$, respectively, while those following 8 weeks of diet consumption were $52.5 \pm 2.3,52.6 \pm 4.9,59.9 \pm 7.5$, $53.3 \pm 4.1$ and $63.8 \pm 2.5 \mathrm{~g}$, respectively, illustrating that the body weight of the PIO+ALO group was significantly increased compared with that of the CD $(\mathrm{P}=0.03)$ and ALO $(\mathrm{P}=0.03)$ groups at the end of the 8-week period (Table II).

Macrovesicular hepatic steatosis areas become small following PIO and/or ALO administration. Following 8 weeks of the dietary treatment, the liver/body weight ratio of the CD group was significantly greater compared with the Control group $(\mathrm{P}=0.006)$. By contrast, no significant differences were identified among the CD, PIO, ALO and PIO+ALO groups (Table II). Mild microvesicular hepatic steatosis was recognized in the Control group and severe macrovesicular hepatic steatosis was recognized in the $\mathrm{CD}$ group. Macrovesicular hepatic steatosis areas in the PIO, ALO and PIO+ALO groups were smaller compared with the CD group (Fig. 1).

$C D$ diet fed to $K K-A^{y}$ mice for 8 weeks does not present evident histological findings of inflammation and fibrosis. No evident histological inflammatory alterations in the liver were observed in the CD group; however, the level of MCP1 expression in the livers of the CD group was significantly increased compared with in the Control group. Conversely, no significant differences were identified between MCP1 expression levels in the livers of the PIO, ALO and PIO+ALO groups compared with in the CD group. There was no evident hepatic fibrosis observed in the CD group and the expression levels of $\alpha \mathrm{SMA}$ in the liver were not significantly different compared with in the Control group (Table III).

Serum parameter of liver tests and metabolic laboratory variables are not altered by PIO or ALO administration. The serum levels of AST $(\mathrm{P}=0.006)$ and ALT $(\mathrm{P}=0.006)$ were significantly higher in the $\mathrm{CD}$ group compared with the Control group, whereas those levels in the PIO, ALO and $\mathrm{PIO}+\mathrm{ALO}$ groups were not statistically different from the CD group (Table IV). Serum adiponectin in the CD group was not statistically different compared with the Control group, while its level in the PIO group demonstrated a tendency to be higher compared with the $\mathrm{CD}$ group $(\mathrm{P}=0.09$; Table IV). Fasting serum insulin in the $\mathrm{CD}$ group demonstrated a tendency to be lower compared with the Control group $(\mathrm{P}=0.055)$, while no significant differences were identified among the $\mathrm{CD}$ diet groups. On the other hand, fasting serum glucose in the $\mathrm{CD}$ group tended to be higher compared with the $\mathrm{PIO}+\mathrm{ALO}(\mathrm{P}=0.082)$ group and HOMA-IR in the PIO+ALO $(\mathrm{P}=0.082)$ group also tended to be lower compared with the CD group (Table IV). Serum TG in the CD group was almost equal to that in the Control group and did not alter with PIO/ALO administration (Table IV). However, serum T-Cho and HDL-C in the CD group were significantly higher compared with the Control group, while serum HDL-C in the PIO+ALO group was higher compared with the ALO group (Table IV).

mRNA expression of CPTla in the liver is significantly increased by ALO administration. The gene expression levels 
Table II. Food intake, body weight and liver weight/body weight at 8 weeks in each group.

\begin{tabular}{lccccc}
\hline Variable & Control & CD & PIO & ALO & PIO+ALO \\
\hline Food intake, kcal/day/body & $23.5 \pm 1.6$ & $16.8 \pm 2.4^{\mathrm{a}}$ & $16.9 \pm 2.3$ & $16.3 \pm 1.5$ & $18.6 \pm 0.7$ \\
Body weight after eight weeks, g & $52.5 \pm 2.3$ & $52.6 \pm 4.9$ & $59.9 \pm 7.5$ & $53.3 \pm 4.1$ & $63.8 \pm 2.5^{\mathrm{b}, \mathrm{c}}$ \\
Liver weight/body weight (\%) & $5.8 \pm 0.6$ & $12.7 \pm 4.4^{\mathrm{a}}$ & $7.2 \pm 1.4$ & $10.6 \pm 2.6$ & $7.3 \pm 2.8$ \\
\hline
\end{tabular}

Data are presented as the mean \pm standard deviation. ${ }^{\mathrm{a}} \mathrm{P}<0.05$ vs. Control group, ${ }^{\mathrm{b}} \mathrm{P}<0.05$ vs. CD group, ${ }^{\mathrm{C}} \mathrm{P}<0.05$ vs. ALO group. CD, choline-deficient diet group; PIO, pioglitazone group; ALO, alogliptin group; PIO+ALO, pioglitazone and alogliptin group.

Table III. mRNA levels of MCP1 and $\alpha$ SMA relative to the mRNA level of GAPDH in the livers of the Control, CD, PIO, ALO and PIO+ALO groups.

\begin{tabular}{lcccrr}
\hline & Control & CD & PIO & ALO & PIO+ALO \\
\hline MCP1 & $0.05 \pm 0.01$ & $0.63 \pm 0.07^{\mathrm{a}}$ & $0.64 \pm 0.04$ & $0.74 \pm 0.08$ & $0.71 \pm 0.14$ \\
$\alpha$ SMA & $1.31 \pm 0.68$ & $2.42 \pm 0.32$ & $1.49 \pm 1.25$ & $1.84 \pm 1.25$ & $1.74 \pm 1.15$ \\
\hline
\end{tabular}

Data are presented as the mean \pm standard deviation. ${ }^{\mathrm{a}} \mathrm{P}<0.05$ vs. Control group. MCP1, monocyte chemoattractant protein $1 ; \alpha \mathrm{SMA}, \alpha$ smooth muscle actin; CD, choline-deficient diet group; PIO, pioglitazone group; ALO, alogliptin group; PIO+ALO, pioglitazone and alogliptin group.
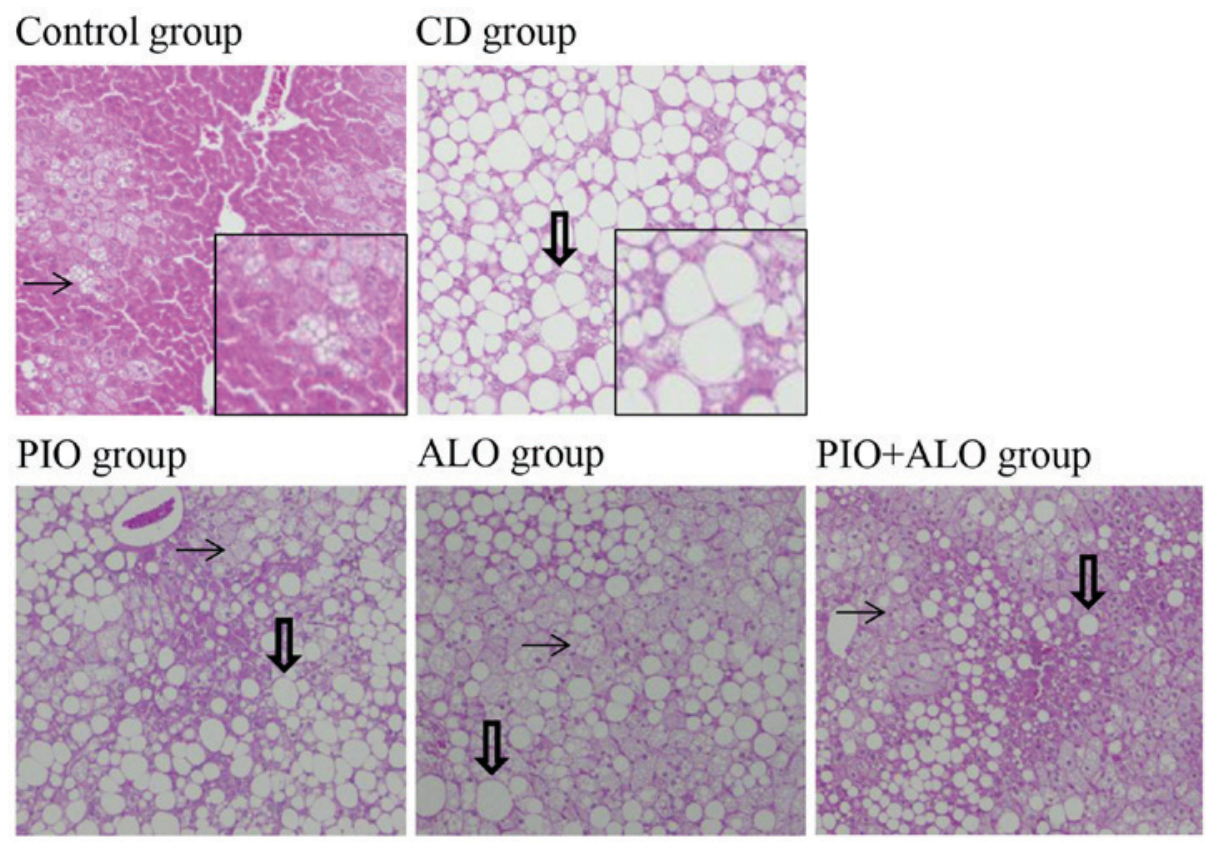

Figure 1. Hematoxylin-eosin staining of livers. Mild microvesicular hepatic steatosis (filled arrow) was recognized in the Control group. Magnification, x40. Severe macrovesicular hepatic steatosis (open arrow) was recognized in the CD group. Magnification, $\mathrm{x} 40$. Enlarged images of microvesicular and macrovesicular steatosis are presented in the lower right of the images of the Control and the CD groups, respectively. Magnification, x100. CD, choline-deficient diet group; PIO, pioglitazone group; ALO, alogliptin group; PIO+ALO, pioglitazone and alogliptin group. Small arrows and open arrows indicate microvesicular steatosis and macrovesicular steatosis respectively.

of enzymes associated with lipid metabolism in liver tissue were examined using RT-qPCR, including genes associated with fatty acid uptake (FATP1, 2, 3 and 4), fatty acid synthesis, fatty acid oxidation (PPAR $\alpha$, L-FABP, CPT1a, AOX and LCAD) and very low density lipoprotein export. The expression levels of FATP3 $(\mathrm{P}=0.006)$ and FAS $(\mathrm{P}=0.006)$ in the liver were significantly increased in the $\mathrm{CD}$ group compared with the Control group (Table V). On the other hand, the expression levels of PPAR $\alpha(\mathrm{P}=0.017)$, L-FABP $(\mathrm{P}=0.018)$, LCAD $(\mathrm{P}=0.011)$ and MTP $(\mathrm{P}=0.006)$ in the liver were significantly decreased in the $\mathrm{CD}$ group compared with the Control group (Table V). Macrovesicular hepatic areas were decreased in the PIO, ALO and PIO+ALO groups compared with the CD group (Fig. 1). The expression levels of lipid metabolic genes in the livers of mice in the PIO, ALO and PIO+ALO groups were also compared with those in the CD 
Table IV. Alterations in serum parameters.

\begin{tabular}{|c|c|c|c|c|c|}
\hline Parameter & Control & $\mathrm{CD}$ & $\mathrm{PIO}$ & ALO & $\mathrm{PIO}+\mathrm{ALO}$ \\
\hline AST, IU/1 & $37 \pm 6$ & $301 \pm 120^{\mathrm{a}}$ & $268 \pm 99$ & $213 \pm 68$ & $236 \pm 76$ \\
\hline ALT, IU/1 & $27 \pm 9$ & $609 \pm 206^{a}$ & $405 \pm 129$ & $463 \pm 204$ & $398 \pm 115$ \\
\hline Adiponectin, mg/ml & $5.50 \pm 2.15$ & $3.62 \pm 1.02$ & $5.03 \pm 0.62$ & $4.10 \pm 0.80$ & $5.40 \pm 1.57$ \\
\hline Fasting serum insulin, $\mathrm{ng} / \mathrm{ml}$ & $3.1 \pm 0.5$ & $1.3 \pm 1.3$ & $0.9 \pm 0.6$ & $1.0 \pm 0.6$ & $0.3 \pm 0.1$ \\
\hline Fasting serum glucose, $\mathrm{mmol} / \mathrm{l}$ & $11.3 \pm 2.1$ & $14.0 \pm 1.8$ & $11.8 \pm 1.0$ & $13.2 \pm 2.9$ & $10.5 \pm 1.9$ \\
\hline HOMA-IR & $1.61 \pm 0.53$ & $0.83 \pm 0.85$ & $0.46 \pm 0.36$ & $0.63 \pm 0.44$ & $0.16 \pm 0.83$ \\
\hline $\mathrm{TG}, \mathrm{mg} / \mathrm{dl}$ & $143 \pm 21$ & $143 \pm 23$ & $118 \pm 24$ & $140 \pm 23$ & $110 \pm 12$ \\
\hline T-Chol, mg/dl & $95 \pm 11$ & $155 \pm 23^{a}$ & $163 \pm 32$ & $141 \pm 29$ & $156 \pm 14$ \\
\hline HDL-C, mg/dl & $64 \pm 8$ & $84 \pm 8^{\mathrm{a}}$ & $85 \pm 13$ & $79 \pm 6$ & $94 \pm 7^{b}$ \\
\hline
\end{tabular}

Data are presented as the mean \pm standard deviation. ${ }^{\mathrm{a}} \mathrm{P}<0.05$ vs. Control group; ${ }^{\mathrm{b}} \mathrm{P}<0.05$ vs. ALO group. $\mathrm{CD}$, choline-deficient diet group; PIO, pioglitazone group; ALO, alogliptin group; PIO+ALO, pioglitazone and alogliptin group; AST, aspartate aminotransferase; ALT, alanine aminotransferase; HOMA-IR, homeostasis model of assessment-insulin resistance; TG, triglyceride; T-Chol, total cholesterol; HDL-C, high density lipoprotein-cholesterol.

Table V. mRNA levels of various target genes/mRNA level of GAPDH in liver following pioglitazone and/or alogliptin administration.

\begin{tabular}{|c|c|c|c|c|c|}
\hline Target gene & Control & $\mathrm{CD}$ & $\mathrm{PIO}$ & ALO & $\mathrm{PIO}+\mathrm{ALO}$ \\
\hline FATP1 & $0.79 \pm 0.14$ & $0.56 \pm 0.17$ & $0.62 \pm 0.12$ & $0.42 \pm 0.13$ & $0.43 \pm 0.14$ \\
\hline FATP2 & $2.64 \pm 0.33$ & $1.25 \pm 0.39^{\mathrm{a}}$ & $1.88 \pm 0.65$ & $1.65 \pm 0.59$ & $1.57 \pm 0.69$ \\
\hline FATP3 & $0.37 \pm 0.05$ & $0.91 \pm 0.26^{\mathrm{a}}$ & $0.72 \pm 0.16$ & $0.78 \pm 0.31$ & $0.82 \pm 0.22$ \\
\hline FATP4 & $0.79 \pm 0.14$ & $0.56 \pm 0.17$ & $0.68 \pm 0.22$ & $0.81 \pm 0.14$ & $0.82 \pm 0.14$ \\
\hline FAS & $0.39 \pm 0.04$ & $0.78 \pm 0.23^{\mathrm{a}}$ & $0.48 \pm 0.10$ & $0.55 \pm 0.13$ & $0.53 \pm 0.15$ \\
\hline L-FABP & $1.21 \pm 0.35$ & $0.78 \pm 0.18^{\mathrm{a}}$ & $1.07 \pm 0.46$ & $0.89 \pm 0.18$ & $0.94 \pm 0.12$ \\
\hline $\mathrm{AOX}$ & $1.30 \pm 0.39$ & $1.03 \pm 0.23$ & $1.64 \pm 0.63$ & $1.16 \pm 0.25$ & $1.45 \pm 0.58$ \\
\hline LCAD & $1.61 \pm 0.44$ & $0.90 \pm 0.25^{\mathrm{a}}$ & $0.93 \pm 0.59$ & $0.92 \pm 0.21$ & $0.91 \pm 0.13$ \\
\hline $\operatorname{PPAR} \alpha$ & $1.25 \pm 0.39$ & $0.72 \pm 0.25^{\mathrm{a}}$ & $1.15 \pm 0.56$ & $0.92 \pm 0.07$ & $1.05 \pm 0.28$ \\
\hline SOD1 & $0.93 \pm 0.45$ & $0.34 \pm 0.14^{\mathrm{a}}$ & $0.57 \pm 0.31$ & $0.55 \pm 0.20$ & $0.70 \pm 0.14$ \\
\hline CPT1a & $0.47 \pm 0.19$ & $0.55 \pm 0.20$ & $0.49 \pm 0.26$ & $2.00 \pm 0.63^{\mathrm{b}, \mathrm{c}}$ & $1.94 \pm 0.73^{\mathrm{b}, \mathrm{c}}$ \\
\hline MTP & $1.39 \pm 0.14$ & $0.94 \pm 0.11^{\mathrm{a}}$ & $0.99 \pm 0.22$ & $0.97 \pm 0.14$ & $0.99 \pm 0.34$ \\
\hline
\end{tabular}

Data are presented as the mean \pm standard deviation. ${ }^{a} \mathrm{P}<0.05$ vs. Control group; ${ }^{\text {}} \mathrm{P}<0.05$ vs. $\mathrm{CD}$ group; ${ }^{\mathrm{C}}<<0.05$ vs. $\mathrm{PIO}$ group; ${ }^{\mathrm{d}} \mathrm{P}<0.05 \mathrm{vs}$. ALO group. CD, choline-deficient diet group; PIO, pioglitazone group; ALO, alogliptin group; PIO+ALO, pioglitazone and alogliptin group; FATP, fatty acid transport protein; FAS, fatty acid synthase; L-FABP, long-chain acyl-coenzyme A dehydrogenase; AOX, acyl-coenzyme A oxidase 1; LCAD, long-chain acyl-coenzyme A dehydrogenase; PPAR $\alpha$, peroxisome proliferator-activated receptor $\alpha$; SOD1, superoxide dismutase 1; CPT1a, carnitine palmitoyltransferase 1a; MTP, microsomal triglyceride transfer protein.

group to examine the mechanism of prevention of fatty liver by PIO and/or ALO administration. The expression of FAS in the liver demonstrated a tendency to be decreased in the PIO group compared with the CD group $(\mathrm{P}=0.051)$, while the expression of CPT1a in the ALO group was significantly increased compared with the $\mathrm{CD}(\mathrm{P}=0.031)$ and $\mathrm{PIO}(\mathrm{P}=0.031)$ groups, whereas the expression of AOX did not alter following administration of PIO and/or ALO. The antioxidant enzyme SOD1 was additionally examined. Its expression in the liver was significantly decreased in the $\mathrm{CD}$ group compared with the Control group $(\mathrm{P}=0.045)$, while it demonstrated a tendency to increase in the PIO+ALO group compared with the CD group $(\mathrm{P}=0.051)$ (Table $\mathrm{V})$.
ALO upregulates CPTla expression via activation of AMP-activated protein kinase. Western blotting demonstrated that ALO promoted the phosphorylation of AMPK on Thr-172 in the livers of the ALO group mice compared with the CD group (Fig. 2).

\section{Discussion}

The present study demonstrated that severe hepatic steatosis developed in $\mathrm{KK}-\mathrm{A}^{\mathrm{y}}$ mice administered a CD diet (the CD group), while weight gain was the same as those fed a standard diet (the Control group). It was concluded that the increased expression levels of FATP3 and FAS, and the decreased expression levels 

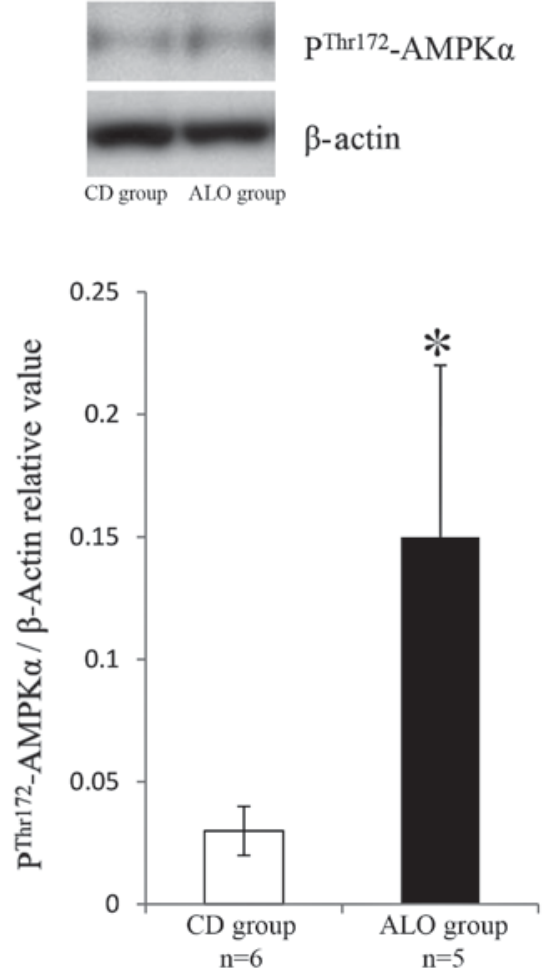

Figure 2. Western blotting of hepatic Thr-172 P-AMPK $\alpha$. Western blotting findings demonstrated that ALO promoted the phosphorylation of AMPK on Thr-172 in the livers of the ALO group mice, compared with the CD group. Band densitometry demonstrated that the bands were denser in the ALO group mice $(n=5)$ compared with the CD group $(n=6)$. Data are expressed as the mean \pm standard error of the mean. ${ }^{*} \mathrm{P}<0.01$ vs. $\mathrm{CD}$ group. AMPK, AMP-activated protein kinase; $\mathrm{CD}$, choline-deficient diet group; PIO, pioglitazone group; ALO, alogliptin group; PIO+ALO, pioglitazone and alogliptin group; P, phosphorylated.

of PPAR $\alpha$, L-FABP, LCAD and MTP, in the livers of the CD group mice contributed to severe hepatic steatosis. MTP is a peptide that incorporates triglycerides into very low density lipoprotein (13), thus it was hypothesized that downregulation of MTP expression contributes to hepatic steatosis. As for glucose metabolism, no significant differences were identified between fasting serum glucose, fasting serum insulin and HOMA-IR levels in the Control and CD groups, indicating that the CD diet did not attenuate insulin resistance or glucose intolerance. By contrast, a methionine- and choline-deficient diet fed to KK-A mice for 8 weeks resulted in severe liver steatosis, inflammatory cell infiltration and liver fibrosis (14).

In the present study, hepatic steatosis was evidently alleviated by administration of PIO. PIO is a TZD, which are known to be effective for T2DM treatment. A previous meta-analysis identified that PIO improves insulin sensitivity, serum ALT levels and histological features in patients with NASH (15). Gastaldelli et al (16) evaluated the association between alterations in plasma adiponectin in patients with NASH and metabolic/histological improvement following treatment with PIO, and concluded that PIO increases the level of adiponectin in patients with NASH, which is important to reverse insulin resistance and improve liver histology. The present study demonstrated that PIO administration in NAFLD model mice with diabetes mellitus tended to increase the plasma concentration of adiponectin. Although the level of adiponectin in the CD group demonstrated a decreasing tendency compared with the Control group, PIO tended to increase the level in the PIO group compared with the CD group. Furthermore, the RT-qPCR analysis of hepatic lipid metabolism demonstrated that PIO decreased the gene expression level of FAS. These results suggested that PIO improved hepatic steatosis by increasing the level of adiponectin and decreasing fatty acid synthesis. Although PIO has been reported to reduce the level of $\alpha$ SMA mRNA (17), the results of the present study did not demonstrate such a reduction in the livers of mice in the PIO and PIO+ALO groups.

A number of DPP-4 inhibitors have been reported to be effective for NASH. Linagliptin was demonstrated to alleviate hepatic steatosis and increased the expression of acetyl-CoA carboxylase 2 in the livers of NASH model mice (18). Kern et al (19) reported that linagliptin significantly decreased the mRNA expression levels of genes associated with inflammation, insulin resistance and fatty acid synthesis in the livers of diet-induced NASH model mice, although such an effect was not observed on the mRNA expression of genes involved in fatty acid oxidation. MK0626, a DPP-4 inhibitor similar to sitagliptin in regard to pharmacokinetics, rescued western diet-induced decreases in hepatic PPAR $\gamma$ coactivator-1 $\alpha$ (PGC-1 $\alpha$ ) and CPT1a mRNA expression (20). In the present study, ALO alleviated hepatic steatosis in CD-diet fed KK-A $\mathrm{A}^{\mathrm{y}}$ mice and significantly increased the hepatic mRNA expression of CPT1a compared with the CD and PIO groups. It was hypothesized that ALO attenuated hepatic steatosis primarily via a fatty acid oxidation pathway, as hepatic mRNA expression is associated with the hepatic uptake of free fatty acid (FATP1-4), and fatty acid synthesis and triacylglycerol export did not alter with ALO administration.

The present study additionally demonstrated that ALO increased hepatic CPT1a expression via the AMPK pathway. CPT1a is important for limiting fatty acid oxidation, and its activity in the liver was identified to be protecting obese mice against hepatic steatosis and insulin resistance (21). Another study demonstrated that GLP-1 reduces hepatic lipogenesis via activation of AMPK (22). AMPK is an evolutionarily conserved metabolic stress-sensing kinase that regulates cellular energy status through phosphorylation of key substrates (23). An in vitro study identified that activation of AMPK increases the expression of CPT1a (24). Dahlhoff et al (25) demonstrated that methyl-donor supplementation (MDS) in obese mice prevented the progression of NAFLD, and suggested that MDS activates AMPK and increases CPT1a expression in the liver, resulting in the promotion of fatty acid oxidation. In the present study, ALO increased hepatic CPT1a expression in CD-diet fed KK-A $\mathrm{A}^{\mathrm{y}}$ mice, which it was hypothesized occurred via AMPK activation, including from MDS. The results of the present study demonstrated that ALO promoted the Thr172 phosphorylation of AMPK and increased CPT1a expression in the livers of CD-diet fed KK- $\mathrm{A}^{\mathrm{y}}$ mice.

The present study has certain limitations. The mRNA expression levels of genes associated with lipid metabolism in liver tissue were examined, although not those of proteins. Furthermore, only a single mouse model was utilized. It may be necessary to examine whether gene expression levels at the mRNA level are the same as at the protein level. In addition, other mouse models may be used in future studies. 
In conclusion, PIO prevented hepatic steatosis by decreasing the mRNA expression level of genes previously reported to be associated with fatty acid synthesis. It is suggested that ALO prevents hepatic steatosis in $\mathrm{KK}-\mathrm{A}^{\mathrm{y}}$ mice fed a CD diet, primarily by increasing the expression of CPTla via the AMPK pathway.

\section{Acknowledgements}

We thank Rika Tohma, and Keiko Masuzaki (Shimane University, Japan) for their technical assistance, as well as the members of our laboratory for the helpful discussion.

\section{Funding}

No funding was received.

\section{Availability of data and materials}

All data generated or analyzed during this study are included in this published article.

\section{Authors' contributions}

HT and SS designed the research study and wrote the paper. TY, TM and NI performed data acquisition and analysis. SI and YK provided major contributions to the design of the study. All authors read and approved the final manuscript.

\section{Ethics approval and consent to participate}

The present study was performed in strict accordance with the recommendations in the Guide for the Care and Use of Laboratory Animals of the National Institutes of Health (Bethesda, MD, USA). The protocol was approved by the Institute for Animal Experimentation of Shimane University (Shimane, Japan; protocol no. IZ23-164).

\section{Consent for publication}

Not applicable.

\section{Competing interests}

The authors declare that they have no competing interests.

\section{References}

1. Bellentani S: The epidemiology of non-alcoholic fatty liver disease. Liver Int 1: 81-84, 2017.

2. Dai W, Ye L, Liu A, Wen SW, Deng J, Wu X and Lai Z: Prevalence of nonalcoholic fatty liver disease in patients with type 2 diabetes mellitus: A meta-analysis. Medicine (Baltimore) 96: e8179, 2017.

3. Angulo P, Kleiner DE, Dam-Larsen S, Adams LA, Bjornsson ES, Charatcharoenwitthaya P, Mills PR, Keach JC, Lafferty HD, Stahler A, et al: Liver fibrosis, but no other histologic features, is associated with long-term outcomes of patients with nonalcoholic fatty liver disease. Gastroenterology 149: 389-397, 2015.

4. Rafig N, Bai C, Fang Y, Srishord M, McCullough A, Gramlich T and Younossi ZM: Long-term follow-up of patients with nonalcoholic fatty liver. Clin Gastroenterol Hepatol 7: 234-238, 2009.

5. Sanyal AJ, Chalasani N, Kowdley KV, McCullough A, Diehl AM, Bass NM, Neuschwander-Tetri BA, Lavine JE, Tonascia J, Unalp A, et al: Pioglitazone, vitamin E, or placebo for nonalcoholic steatohepatitis. N Engl J Med 362: 1675-1685, 2010.
6. Da Silva Morais A, Lebrun V, Abarca-Quinones J, Brichard S, Hue L, Guigas B, Viollet B and Leclercq IA: Prevention of steatohepatitis by pioglitazone: Implication of adiponectin-dependent inhibition of SREBP-1c and inflammation. J Hepatol 50: 489-500, 2009.

7. Yilmaz Y, Yonal O, Deyneli O, Celikel CA, Kalayci C and Duman DG: Effects of sitagliptin in diabetic patients with nonalcoholic steatohepatitis. Acta Gastroenterol Belg 75: 240-244, 2012.

8. Mashitani T, Noguchi R, Okura Y, Namisaki T, Mitoro A, Ishii H, Nakatani T, Kikuchi E, Moriyasu H, Matsumoto M, et al: Efficacy of alogliptin in preventing non-alcoholic fatty liver disease progression in patients with type 2 diabetes. Biomed Rep 4: 183-187, 2016.

9. Nonogaki K, Nozue K and Oka Y: Social isolation affects the development of obesity and type 2 diabetes in mice. Endocrinology 148: 4658-4666, 2007.

10. Haluzik MM, Lacinova Z, Dolinkova M, Haluzikova D, Housa D, Horinek A, Vernerova Z, Kumstyrova T and Haluzik M: Improvement of insulin sensitivity after peroxisome proliferator-activated receptor-alpha agonist treatment is accompanied by paradoxical increase of circulating resistin levels. Endocrinology 147: 4517-4524, 2006.

11. Rinella ME, Elias MS, Smolak RR, Fu T, Borensztajn J and Green RM: Mechanisms of hepatic steatosis in mice fed a lipogenic methionine choline-deficient diet. J Lipid Res 49: 1068-1076, 2008.

12. Rozen S and Skaletsky H: Primer3 on the WWW for general users and for biologist programmers. Methods Mol Biol 132: 365-386, 2000.

13. Raabe M, Véniant MM, Sullivan MA, Zlot CH, Björkegren J, Nielsen LB, Wong JS, Hamilton RL and Young SG: Analysis of the role of microsomal triglyceride transfer protein in the liver of tissue-specific knockout mice. J Clin Invest 103: 1287-1298, 1999.

14. Okumura K, Ikejima K, Kon K, Abe W, Yamashina S, Enomoto N, Takei Y and Sato N: Exacerbation of dietary steatohepatitis and fibrosis in obese, diabetic KK-A(y) mice. Hepatol Res 36: 217-228, 2006.

15. Boettcher E, Csako G, Pucino F, Wesley R and Loomba R: Meta-analysis: Pioglitazone improves liver histology and fibrosis in patients with non-alcoholic steatohepatitis. Aliment Pharmacol Ther 35: 66-75, 2012.

16. Gastaldelli A, Harrison S, Belfort-Aguiar R, Hardies J, Balas B, Schenker S and Cusi K: Pioglitazone in the treatment of NASH: The role of adiponectin. Aliment Pharmacol Ther 32: 769-775, 2010.

17. Kon K, Ikejima K, Hirose M, Yoshikawa M, Enomoto N, Kitamura T, Takei Y and Sato N: Pioglitazone prevents early-phase hepatic fibrogenesis caused by carbon tetrachloride. Biochem Biophys Res Commun 291: 55-61, 2002.

18. Klein T, Fujii M, Sandel J, Shibazaki Y, Wakamatsu K, Mark M and Yoneyama H: Linagliptin alleviates hepatic steatosis and inflammation in a mouse model of non-alcoholic steatohepatitis. Med Mol Morphol 47: 137-149, 2014.

19. Kern M, Klöting N, Niessen HG, Thomas L, Stiller D, Mark M, Klein T and Blüher M: Linagliptin improves insulin sensitivity and hepatic steatosis in diet-induced obesity. PLoS One 7: e38744, 2012.

20. Aroor AR, Habibi J, Ford DA, Nistala R, Lastra G, Manrique C, Dunham MM, Ford KD, Thyfault JP, Parks EJ, et al: Dipeptidyl peptidase-4 inhibition ameliorates Western diet-induced hepatic steatosis and insulin resistance through hepatic lipid remodeling and modulation of hepatic mitochondrial function. Diabetes 64 : 1988-2001, 2015.

21. Orellana-Gavaldà JM, Herrero L, Malandrino MI, Pañeda A, Sol Rodríguez-Peña M, Petry H, Asins G, Van Deventer S, Hegardt FG and Serra D: Molecular therapy for obesity and diabetes based on a long-term increase in hepatic fatty-acid oxidation. Hepatology 53: 821-832, 2011.

22. Ben-Shlomo S, Zvibel I, Shnell M, Shlomai A, Chepurko E, Halpern Z, Barzilai N, Oren R and Fishman S: Glucagon-like peptide-1 reduces hepatic lipogenesis via activation of AMP-activated protein kinase. J Hepatol 54: 1214-1223, 2011.

23. Steinberg GR and Kemp BE: AMPK in health and disease. Physiol Rev 89: 1025-1078, 2009.

24. Kim JH, Kang SI, Shin HS, Yoon SA, Kang SW, Ko HC and Kim SJ: Sasa quelpaertensis and p-coumaric acid attenuate oleic acid-induced lipid accumulation in HepG2 cells. Biosci Biotechnol Biochem 77: 1595-1598, 2013.

25. Dahlhoff C, Worsch S, Sailer M, Hummel BA, Fiamoncini J, Uebel K, Obeid R, Scherling C, Geisel J, Bader BL and Daniel H: Methyl-donor supplementation in obese mice prevents the progression of NAFLD, activates AMPK and decreases acyl-carnitine levels. Mol Metab 3: 565-580, 2014. 